\title{
An Approach to Design a Simple Human Detecting Robot for Cost Effective Home Security System as well as Various Rescue Missions
}

\author{
Sabyasachi Bhowmick \\ B.Tech (ECE); Director \\ Quantatrack Technology Pvt. Ltd. \\ Agartala, Tripura (West), India
}

\begin{abstract}
In this wonderful world, various robots are being made for various purposes. Every robot circuit performs individual tasks assigned to it. In this paper, a simple Robot is designed to detect and to inform the presence of human with a new approach. In this system, inputs are taken through an Ultrasonic Sensor, a PIR sensor and an array of IR Sensors. Ultrasonic and IR Sensors are there to detect the path and direct the Robot and PIR Sensor is to detect Human presence. $\mathrm{RF}$ transmitter is present to transmit the information of human presence and through the receiver circuit, information is received. After receiving the information, the receiver immediately gives output which turns the buzzer/ alarm and the display ON. A program has been written and executed for the said purpose using AVR Microcontroller. The hardware implementation is made quite simply and cost effectively. The experiments are observed deeply and the results show that the system provides an efficient way for the said purpose.
\end{abstract}

\section{Keywords}

PIR Sensor, SR-04, HT12E, HT12D, LM35. Mobile ROBOT, Motion Detection, Rescue

\section{INTRODUCTION}

Robotic systems for Human detection is an excellent innovation of modern technology. It provides an outstanding service to the mankind. Human detection has always been a matter of great curiosity to the people. There are different ways to detect a human being. The three most popular methods are 1. Image Capturing through a Camera/Image Sensor, 2. Using PIR Sensor and 3. Using Proximity Sensor. First one is more costly than rest of the two. The last one is bulkier than the second one. The Simplest and Cost effective method is using a PIR Sensor. Through this detection mechanism human can be detected easily and accurately. Again, there are various methods of information transmission between two devices. RF transmission is one of the cheapest and simplest method, where signals can be transmitted and received easily through the Transmitter and Receiver Module. Therefore RF transmission has been used in this system.

This paper proposes a mobile robotic system which is able to work in extremely calamitous conditions. The proposed system uses a PIR sensor to detect the motion of human body and combination of IR sensor pairs and Ultrasonic Sensor to detect any obstacle on the way and to choose the path of robot. Having detected the sign of living humans, the system transmits the information and in the receiver part we can find display the status as well as turn a buzzer ON.
The existing system has suffered many problems like high cost for setting up communication between robot and rescue control unit, noisy wireless communication link between robot and control unit which ultimately stopped robot to function etc. The proposed system is able to solve all these problems.

\section{COMPONENTS USED}

The proposed system has used various components to do the task appropriately. The sensors and other components are placed in such way so that they can detect the human and transmit the information easily and move smoothly. Different components, used in this system, are,

Microcontroller (ATmega32), PIR Sensor, Ultrasonic Sensor, IR Sensor array, RF Transmitter and Receiver, LCD Display, Buzzer, DC motors etc.

\section{THE SYSTEM}

Our proposed system is a system which moves forward on the basis of an Ultrasonic Sensor module. Ultrasonic Sensor calculates the distance between the robot and the obstacle, and gives output to the motor driver through Microcontroller. It is programmed such that $11 \mathrm{~cm}$ distance is set as the threshold distance for the Ultrasonic Sensor to stop. If there is any obstacle detected by the Ultrasonic Sensor, it will give input to the microcontroller which turns on the Right IR sensor pair, which will give command to the motor driver as 'Turn Right'. After turning in the right side the Ultrasonic Sensor will again calculate the distance to move forward. If again there is any obstacle found, the Ultrasonic Sensor will give output to the motor driver(through MCU) as 'Turn left', i.e. 'Reset previous position', and then Left IR Sensor pair will be activated and the vehicle will Turn Left from its actual position, when the Ultrasonic Sensor will calculate the distance again. If any obstacle is found again, then again the Ultrasonic Sensor will give output as 'Turn Left' which is nothing but turning 180 degrees from the actual position. Every operation will be done through Microcontroller. Thus the movement operation is done.

With the help of LM35 (temperature sensor), the range of PIR sensor is set as $4 \mathrm{ft}$. Therefore the PIR Sensor senses human within $4 \mathrm{ft}$. Whenever a human is detected by the sensor, it immediately stops the robot on that spot and gives output to the RF transmitter which transmits this information and the receiver receives it. After receiving the signal, the receiver gives the output to anther Microcontroller which turns the Alarm system and the Display ON. (A flashlight can also be connected with the receiver if the system is made for home security purpose). 


\subsection{ATmega32}

Microcontrollers are used as a brain for these types of operations. Microcontrollers have inbuilt memory, input and output pins through which tasks become easier. Here, ATmega32 microcontroller from AVR family is used. The controller is well known for its internal $8 \mathrm{MHz}$ clock frequency, Advanced RISC architecture, High endurance Nonvolatile memory segments, Four PWM channels, Programmable Serial USART, Master/Slave SPI Serial Interface, Programmable Watchdog Timer with Separate Onchip Oscillator and many more features. Here BERD V.1.0 (Basic Electronics and Robotics Development) by Riscin Technologies Pvt. Ltd. is used as ATmega32 development board.

\subsection{Ultrasonic Sensor}

The ultrasonic sensor is used to calculate distance. Here Ultrasonic Sensor module 'SR-04' has been used. The transmitter part transmits ultrasonic wave and the receiver part receives it. The ultrasonic sensor output signal is fed to the microcontroller ATMEGA32 via the serial interface. A suitable embedded 'c' program is written in the algorithm here to take $11 \mathrm{~cm}$ distance as its threshold and help the Robot in moving forward.

\subsection{PIR Sensor}

The $R B-P l x-75$ from Parallax is a PIR sensor which is made for short range operations. With the help of a temperature sensor (LM35) the range has been extended up to $4 \mathrm{ft}$. The PIR sensor resets itself when nothing is detected. This feature is a big advantage of the said sensor. The output of the PIR sensor is a pulse of $0 \mathrm{~V}$ when it is in idle mode and $5 \mathrm{~V}$ when any human is detected. If something is detected in multiple times, the sensor will give $5 \mathrm{~V}$ output constantly till then the human is present there. This pyro-electric device has three different pins, namely Vcc Pin, Ground Pin and Data Pin (Output Pin).

\subsection{IR Sensor Array}

IR Sensor Array consists of three sections-transmitter and receiver. Transmitter continuously sends the IR signal and receiver receives the reflected light from the obstacle. So, it has been used so that the obstacle detection operation is done more accurately in the proposed system. To get precise output, LM339 comparator has been used. Whenever receiver receives reflected IR signal, LED glows to show that obstacle is detected on its path.

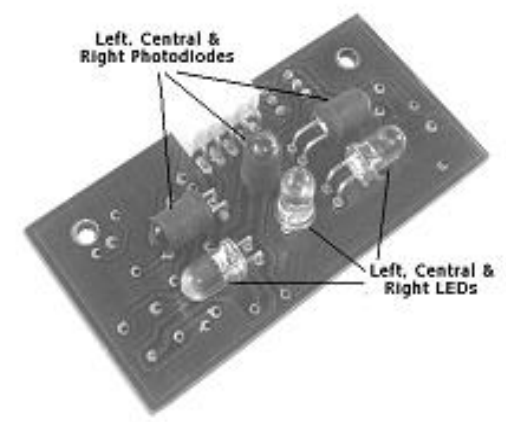

Fig 1: IR Sensor Array

\subsection{RF Transmitter}

HT12E (18 pin DIP) CMOS LSI encoder is used for information transmission of the robot. Encoder is capable of encoding 12 bits of information ( 4 data bits $\& 8$ address bits). High noise in mainly easy interface and low standby current are some of its features. Its operating voltage is $2.4-12 \mathrm{~V}$. It has also got built-in oscillator. A wired antenna is used for transmission of encoded signals serially. RF frequency used for transmission is $433.92 \mathrm{MHz}$

\subsection{RF Receiver}

HT12D (18 pin DIP) CMOS LSI decoder is used for receiving signals from the transmitter. It decodes 4 bits of data sent by encoder and output is given to the controller. The receiver is capable of decoding 12 bits of data. Its operating voltage is 2.4-12 V. A wired antenna is used to receive the encoded signal serially. It is compatible with HT12E.

\subsection{LCD \& Alarm}

The $16 \times 2$ LCD is an LCD module having 16 pins, 16 rows and 2 columns of display messages. The $16 \times 2$ module is used widely in most of the robotic circuits to display the messages. The features are given below:

$$
\begin{aligned}
& \cdot 5 \times 8 \text { dots with cursor } \\
& \text { - Built-in controller (KS } 0066 \text { or Equivalent) } \\
& \cdot+5 \mathrm{~V} \text { power supply (Also available for }+3 \mathrm{~V} \text { ) } \\
& \text { - } 1 / 16 \text { duty cycle } \\
& \text { - B/L to be driven by pin 1, pin } 2 \text { or pin } 15 \text {, pin } 16 \\
& \text { or A.K (LED) } \\
& \text { - N.V. optional for }+3 \mathrm{~V} \text { power supply }
\end{aligned}
$$

A buzzer is connected as the Audio Alarm. The buzzer's specification is F/BC 35 .

\section{BLOCK DIAGRAM}
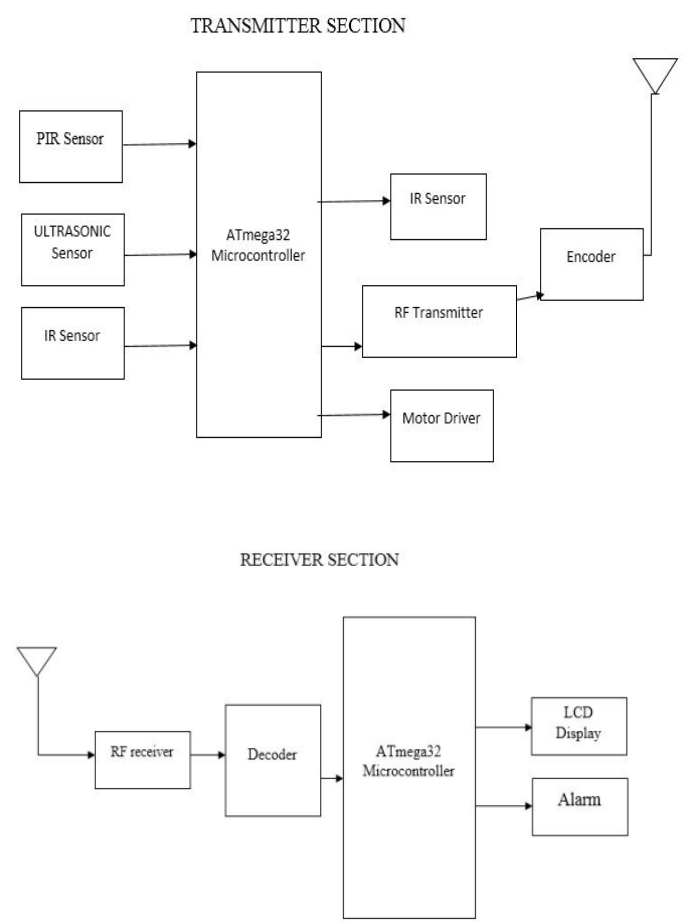

Fig 2: Block diagram of Tx \& Rx 


\section{RESULTS \& DISCUSSIONS}

To make the prototype of the proposed system, sensors had been assembled as mentioned. Two DC motors were connected with wheels, and one castor wheel was connected to help the ROBOT in navigation. $9 \mathrm{~V}$ battery was connected as power source. Then many obstacles were placed in the path and the ROBOT detected those obstacles successfully and the Ultrasonic Sensor was able to detect its path every time. At the second part when a human was within the range of $4 \mathrm{ft}$. of the PIR Sensor, it stopped the ROBOT and started generating output and turning the RF transmitter ON. On the other hand, the RF receiver received the transmitted signal showing an output to the LCD as 'Human Detected'.

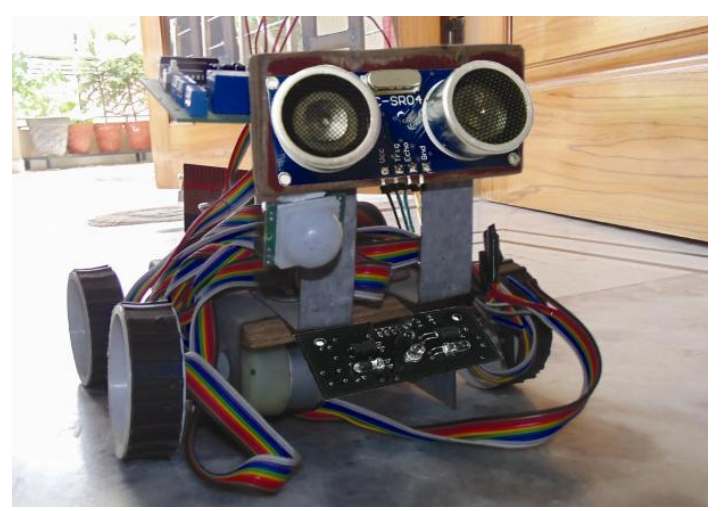

Fig 3: Prototype of the proposed system

\section{CONCLUSION \& FUTURE SCOPE}

The main purpose of the proposed system to detect human beings and give us information about the presence of human beings in a cost effective mechanism. Sometimes, it is not feasible for rescue personnel to individually visit the site (war field, earthquake-stricken area, mines etc.) and check who is alive and who needs rescue. So, in such circumstances, the proposed system can be of great importance. It can be deployed to detect alive human beings and send the information regarding the situation of the spot to rescue team for proceeding further rescue operation. Furthermore, it can be implemented easily as a 'Low cost Home Security System'.

The prototype can be further enhanced in the future by adding a Camera to capture the image of the human, from which we can guess if he/she/they are alive or not. In addition, to know the exact location, GPS system can be implemented. For increasing the range of communication with the rescue team, GSM module can be included. Furthermore, metal and bomb detectors can be used to protect from possible damage. Lightweight solar panel can be included to make the robot 'Self Charging'.

\section{REFERENCES}

[1] Ying-Wen Bai, Li-Sih Shen and Zong-Han Li "Design and Implementation of an Embedded Home Surveillance System by Use of Multiple Ultrasonic Sensors" IEEE Transactions on Consumer Electronics, Vol. 56, No. 1, February 2010, PP. 119-124

[2] Rufaida Shamroukh, Fahed Awad "Detection of surviving humans in destructed environments using a simulated autonomous robot "IEEE Transaction, March 24-26, 2009.

[3] Pissokas, John and Malcolm, Chris (2001) "Experiments with Sensors for Urban Search and Rescue Robots", Dept. of Computer Science, University of Essex and Division of Informatics, University of Edinburgh, Scotland UK.

[4] A Survey of Human-Sensing: Methods for Detecting Presence, Count, Location, Track, and Identity By THIAGO TEIXEIRA, Yale University; GERSHON DUBLON, Massachusetts Institute of Technology And ANDREAS SAVVIDES, Yale University

[5] Burion, Steve (2004), Casper, J. (2002), Amerada, Yams, Igarashit, \& Matsunos, (2004) and Cappello, C., etal (2005) "Human Detection for Robotic Urban Search and Rescue" Carnegie Mellon University, Institute of Production Robotique (IPR) LSRO2 - VRAI-Group, Microtechnique, February 2004.

[6] http://en.wikipedia.org/wiki/Sensors.

\section{BIOGRAPHIES}

Mr. Sabyasachi Bhowmick is a B.Tech in Electronics and Communication Engineering and is the Director of Self owned company named as QUANTATRACK Technology Pvt. Ltd, Agartala, Tripura (West), India. 